\title{
Existence and Uniqueness of Positive Solutions of Boundary-Value Problems for Fractional Differential Equations with $p$-Laplacian Operator and Identities on the Some Special Polynomials
}

\author{
Erdoğan Şen, ${ }^{1,2}$ Mehmet Acikgoz, ${ }^{3}$ Jong Jin Seo, ${ }^{4,5}$ Serkan Araci, ${ }^{6}$ and Kamil Oruçoğlu' ${ }^{2}$ \\ ${ }^{1}$ Department of Mathematics, Faculty of Science and Letters, Namik Kemal University, 59030 Tekirdăg, Turkey \\ ${ }^{2}$ Department of Mathematics Engineering, Istanbul Technical University, Maslak, 34469 Istanbul, Turkey \\ ${ }^{3}$ Department of Mathematics, Faculty of Science and Arts, University of Gaziantep, 27310 Gaziantep, Turkey \\ ${ }^{4}$ Department of Applied Mathematics, Pukyong National University, Busan 608-737, Republic of Korea \\ ${ }^{5}$ Department of Mathematics, College of Natural Sciences, Kwangwoon University, Seoul 139-701, Republic of Korea \\ ${ }^{6}$ Atatürk Street, 31290 Hatay, Turkey
}

Correspondence should be addressed to Serkan Araci; mtsrkn@hotmail.com

Received 13 May 2013; Accepted 28 September 2013

Academic Editor: Josip E. Pečarić

Copyright (C) 2013 Erdoğan Şen et al. This is an open access article distributed under the Creative Commons Attribution License, which permits unrestricted use, distribution, and reproduction in any medium, provided the original work is properly cited.

We consider the following boundary-value problem of nonlinear fractional differential equation with $p$-Laplacian operator $D_{0+}^{\beta}\left(\phi_{p}\left(D_{0+}^{\alpha} u(t)\right)\right)+a(t) f(u)=0,0<t<1, u(0)=\gamma u(h)+\lambda, u^{\prime}(0)=\mu, \phi_{p}\left(D_{0+}^{\alpha} u(0)\right)=\left(\phi_{p}\left(D_{0+}^{\alpha} u(1)\right)\right)^{\prime}=\left(\phi_{p}\left(D_{0+}^{\alpha} u(0)\right)\right)^{\prime \prime}=$ $\left(\phi_{p}\left(D_{0+}^{\alpha} u(0)\right)\right)^{\prime \prime \prime}=0$, where $1<\alpha \leqslant 2,3<\beta \leqslant 4$ are real numbers, $D_{0+}^{\alpha}, D_{0+}^{\beta}$ are the standard Caputo fractional derivatives, $\phi_{p}(s)=|s|^{p-2} s, p>1, \phi_{p}^{-1}=\phi_{q}, 1 / p+1 / q=1,0 \leqslant \gamma<1,0 \leqslant h \leqslant 1, \lambda, \mu>0$ are parameters, $a:(0,1) \rightarrow[0,+\infty)$, and $f:[0,+\infty) \rightarrow[0,+\infty)$ are continuous. By the properties of Green function and Schauder fixed point theorem, several existence and nonexistence results for positive solutions, in terms of the parameters $\lambda$ and $\mu$ are obtained. The uniqueness of positive solution on the parameters $\lambda$ and $\mu$ is also studied. In the final section of this paper, we derive not only new but also interesting identities related special polynomials by which Caputo fractional derivative.

\section{Introduction}

In 1695, L'Hôpital asked Leibniz: what if the order of the derivative is $1 / 2$ ? To which Leibniz considered in a useful means, thus it follows that will be equal to $x \sqrt{d x: x}$, an obvious paradox. In recent years, fractional calculus has been studied by many mathematicians from Leibniz's time to the present.

Also, fractional differential equations arise in many engineering and scientific disciplines as the mathematical modelling of systems and processes in the fields of physics, fluid flows, electrical networks, viscoelasticity, aerodynamics, and many other branches of science. For details, see [1-9].
In the last few decades, fractional-order models are found to be more adequate than integer-order models for some real world problems. Recently, there have been some papers dealing with the existence and multiplicity of solutions (or positive solutions) of nonlinear initial fractional differential equations by the use of techniques of nonlinear analysis [1021], upper and lower solutions method [22-24], fixed point index $[25,26]$, coincidence theory [27], Banach contraction mapping principle [28], and so forth.

Chai [11] investigated the existence and multiplicity of positive solutions for a class of boundary-value problem of fractional differential equation with $p$-Laplacian operator 


$$
\begin{gathered}
D_{0+}^{\beta}\left(\phi_{p}\left(D_{0+}^{\alpha} u(t)\right)\right)+f\left(t, u(t), D_{0+}^{\rho} u(t)\right)=0, \\
0<t<1, \\
u(0)=0, \quad u(1)+\sigma D_{0+}^{\gamma} u(1)=0, \quad D_{0+}^{\alpha} u(0)=0,
\end{gathered}
$$

where $1<\alpha \leq 2,0<\gamma \leq 1,0 \leq \alpha-\gamma-1, \sigma$ is a positive constant number, and $D_{0+}^{\alpha}, D_{0+}^{\beta}, D_{0+}^{\gamma}$ are the standard Riemann-Liouville derivatives. By means of the fixed point theorem on cones, some existence and multiplicity results of positive solutions are obtained.

Although the fractional differential equation boundaryvalue problems have been studied by several authors, very little is known in the literature on the existence and nonexistence of positive solutions of fractional differential equation boundary-value problems with $p$-Laplacian operator when a parameter $\lambda$ is involved in the boundary conditions. We also mention that, there is very little known about the uniqueness of the solution of fractional differential equation boundaryvalue problems with $p$-Laplacian operator on the parameter $\lambda$. Han et al. [29] studied the existence and uniqueness of positive solutions for the fractional differential equation with $p$-Laplacian operator

$$
\begin{aligned}
D_{0+}^{\beta}\left(\phi_{p}\left(D_{0+}^{\alpha} u(t)\right)\right) & +a(t) f(u)=0, \quad 0<t<1, \\
u(0) & =\gamma u(\xi)+\lambda, \\
\phi_{p}\left(D_{0+}^{\alpha} u(0)\right) & =\left(\phi_{p}\left(D_{0+}^{\alpha} u(1)\right)\right)^{\prime} \\
& =\left(\phi_{p}\left(D_{0+}^{\alpha} u(0)\right)\right)^{\prime \prime}=0,
\end{aligned}
$$

where $0<\alpha \leqslant 1,2<\beta \leqslant 3$ are real numbers; $D_{0+}^{\alpha}, D_{0+}^{\beta}$ are the standard Caputo fractional derivatives; $\phi_{p}(s)=|s|^{p-2} s$, $p>1$. Therefore, to enrich the theoretical knowledge of the above, in this paper, we investigate the following $p$-Laplacian fractional differential equation boundary-value problem:

$$
\begin{aligned}
D_{0+}^{\beta}\left(\phi_{p}\left(D_{0+}^{\alpha} u(t)\right)\right)+a(t) f(u)=0, \quad 0<t<1, & u^{\prime}(0)=\mu, \\
u(0) & =\gamma u(h)+\lambda, \quad \phi_{p}\left(D_{0+}^{\alpha} u(0)\right)=\left(\phi_{p}\left(D_{0+}^{\alpha} u(1)\right)\right)^{\prime}=\left(\phi_{p}\left(D_{0+}^{\alpha} u(0)\right)\right)^{\prime \prime} \\
& =\left(\phi_{p}\left(D_{0+}^{\alpha} u(0)\right)\right)^{\prime \prime \prime}=0,
\end{aligned}
$$

where $1<\alpha \leqslant 2,3<\beta \leqslant 4$ are real numbers, $D_{0+}^{\alpha}, D_{0+}^{\beta}$ are the standard Caputo fractional derivatives, $\phi_{p}(s)=|s|^{p-2} s$, $p>1, \phi_{p}^{-1}=\phi_{q}, 1 / p+1 / q=1,0 \leqslant \gamma<1,0 \leqslant h \leqslant 1$, $\lambda, \mu>0$ are parameters, $a:(0,1) \rightarrow[0,+\infty)$, and $f:$ $[0,+\infty) \rightarrow[0,+\infty)$ are continuous. By the properties of Green function and Schauder fixed point theorem, several existence and nonexistence results for positive solutions, in terms of the parameters $\lambda$ and $\mu$ are obtained. The uniqueness of positive solution on the parameters $\lambda$ and $\mu$, is also studied.

\section{Preliminaries and Related Lemmas}

Definition 1 (see [4]). The Riemann-Liouville fractional integral of order $\alpha>0$ of a function $y:(0,+\infty) \rightarrow \mathbb{R}$ is given by

$$
I_{0+}^{\alpha} y(t)=\frac{1}{\Gamma(\alpha)} \int_{0}^{t}(t-s)^{\alpha-1} y(s) d s
$$

provided the right side is pointwise defined on $(0,+\infty)$.

Definition 2 (see [4]). The Caputo fractional derivative of order $\alpha>0$ of a continuous function $y:(0,+\infty) \rightarrow \mathbb{R}$ is given by

$$
D_{0+}^{\alpha} y(t)=\frac{1}{\Gamma(n-\alpha)} \int_{0}^{t} \frac{y^{(n)}(s)}{(t-s)^{\alpha-n+1}} d s,
$$

where $n$ is the smallest integer greater than or equal to $\alpha$, provided that the right side is pointwise defined on $(0,+\infty)$.

Remark 3 (see [8]). By Definition 2, under natural conditions on the function $f(t)$, for $\alpha \rightarrow n$, the Caputo derivative becomes a conventional $n$th derivative of the function $f(t)$.

Remark 4 (see [4]). As a basic example,

$$
\begin{aligned}
D_{0^{+}}^{\alpha} t^{\mu}= & \mu(\mu-1) \cdots(\mu-n+1) \\
& \times \frac{\Gamma(1+\mu-n)}{\Gamma(1+\mu-\alpha)} t^{\mu-\alpha}, \quad \text { for } t \in(0, \infty) .
\end{aligned}
$$

In particular, $D_{0^{+}}^{\alpha} t^{\mu}=0, \mu=0,1, \ldots, n-1$, where $D_{0^{+}}^{\alpha}$ is the Caputo fractional derivative, $n$ is the smallest integer greater than or equal to $\alpha$.

From the definition of the Caputo derivative and Remark 4, we can obtain the following statement.

Lemma 5 (see [4]). Let $\alpha>0$. Then, the fractional differential equation

$$
D_{0+}^{\alpha} u(t)=0
$$

has a unique solution

$$
\begin{array}{r}
u(t)=c_{0}+c_{1} t+c_{2} t^{2}+\cdots+c_{n-1} t^{n-1}, \\
c_{i} \in \mathbb{R}, i=0,1,2, \ldots, n-1,
\end{array}
$$

where $n$ is the smallest integer greater than or equal to $\alpha$.

Lemma 6 (see [4]). Let $\alpha>0$. Assume that $u \in C^{n}[0,1]$. Then

$$
I_{0+}^{\alpha} D_{0+}^{\alpha} u(t)=u(t)+c_{0}+c_{1} t+c_{2} t^{2}+\cdots+c_{n-1} t^{n-1},
$$

for some $c_{i} \in \mathbb{R}, i=0,1,2, \ldots, n-1$, where $n$ is the smallest integer greater than or equal to $\alpha$.

Lemma 7. Let $y \in C[0,1]$ and $1<\alpha \leq 2$. Then, fractional differential equation boundary-value problem

$$
\begin{gathered}
D_{0+}^{\alpha} u(t)=y(t), \quad 0<t<1, \\
u(0)=\gamma u(h)+\lambda, \quad u^{\prime}(0)=\mu
\end{gathered}
$$


has a unique solution

$$
\begin{aligned}
u(t)= & \int_{0}^{t} \frac{(t-s)^{\alpha-1}}{\Gamma(\alpha)} y(s) d s \\
& +\frac{\gamma}{1-\gamma} \int_{0}^{h} \frac{(h-s)^{\alpha-1}}{\Gamma(\alpha)} y(s) d s+\frac{\lambda+\gamma \mu h}{1-\gamma} .
\end{aligned}
$$

Proof. We apply Lemma 6 to reduce (10) to an equivalent integral equation,

$$
u(t)=I_{0+}^{\alpha} y(t)+c_{0}+c_{1} t, \quad c_{0}, c_{1} \in \mathbb{R} .
$$

Consequently, the general solution of (10) is

$$
u(t)=\int_{0}^{t} \frac{(t-s)^{\alpha-1}}{\Gamma(\alpha)} y(s) d s+c_{0}+c_{1} t, \quad c_{0}, c_{1} \in \mathbb{R} .
$$

By (11), we have

$$
c_{0}=\frac{\gamma}{1-\gamma} \int_{0}^{h} \frac{(h-s)^{\alpha-1}}{\Gamma(\alpha)} y(s) d s+\frac{\gamma c_{1} h}{1-\gamma}+\frac{\lambda}{1-\gamma},
$$

and since $u^{\prime}(0)=c_{1}$, we have by (11)

$$
c_{1}=\mu
$$

Therefore, the unique solution of problem (10) and (11) is

$$
\begin{aligned}
u(t)= & \int_{0}^{t} \frac{(t-s)^{\alpha-1}}{\Gamma(\alpha)} y(s) d s \\
& +\frac{\gamma}{1-\gamma} \int_{0}^{h} \frac{(h-s)^{\alpha-1}}{\Gamma(\alpha)} y(s) d s+\frac{\lambda}{1-\gamma}+\frac{\gamma \mu h}{1-\gamma} .
\end{aligned}
$$

Lemma 8. Let $y \in C[0,1]$ and $1<\alpha \leq 2,3<\beta \leq 4$. Then, fractional differential equation boundary-value problem

$$
\begin{gathered}
D_{0+}^{\beta}\left(\phi_{p}\left(D_{0+}^{\alpha} u(t)\right)\right)+y(t)=0, \quad 0<t<1, \\
u(0)=\gamma u(h)+\lambda, \quad u^{\prime}(0)=\mu, \\
\phi_{p}\left(D_{0+}^{\alpha} u(0)\right)=\left(\phi_{p}\left(D_{0+}^{\alpha} u(1)\right)\right)^{\prime}=\left(\phi_{p}\left(D_{0+}^{\alpha} u(0)\right)\right)^{\prime \prime} \\
=\left(\phi_{p}\left(D_{0+}^{\alpha} u(0)\right)\right)^{\prime \prime \prime}=0,
\end{gathered}
$$

has a unique solution

$$
\begin{aligned}
u(t)= & \int_{0}^{t} \frac{(t-s)^{\alpha-1}}{\Gamma(\alpha)} \phi_{q}\left(\int_{0}^{1} H(s, \tau) y(\tau) d \tau\right) d s \\
& +\frac{\gamma}{1-\gamma} \int_{0}^{h} \frac{(h-s)^{\alpha-1}}{\Gamma(\alpha)} \phi_{q}\left(\int_{0}^{1} H(s, \tau) y(\tau) d \tau\right) d s \\
& +\frac{\lambda+\gamma \mu h}{1-\gamma}
\end{aligned}
$$

where

$$
H(t, s)= \begin{cases}\frac{t(\beta-1)(1-s)^{\beta-2}-(t-s)^{\beta-1}}{\Gamma(\beta)}, & 0 \leq s \leq t \leq 1, \\ \frac{t(\beta-1)(1-s)^{\beta-2}}{\Gamma(\beta)}, & 0 \leq t \leq s \leq 1 .\end{cases}
$$

Proof. From Lemma 6, the boundary-value problems (18) and (19) are equivalent to the integral equation

$$
\phi_{p}\left(D_{0+}^{\alpha} u(t)\right)=-I_{0+}^{\beta} y(t)+c_{0}+c_{1} t+c_{2} t^{2}+c_{3} t^{3},
$$

for some $c_{0}, c_{1}, c_{2}, c_{3} \in \mathbb{R}$; that is,

$$
\phi_{p}\left(D_{0+}^{\alpha} u(t)\right)=-\int_{0}^{t} \frac{(t-\tau)^{\beta-1}}{\Gamma(\beta)} y(\tau) d \tau+c_{0}+c_{1} t+c_{2} t^{2}+c_{3} t^{3}
$$

By the boundary conditions $\phi_{p}\left(D_{0+}^{\alpha} u(0)\right)=\left(\phi_{p}\left(D_{0+}^{\alpha} u(1)\right)\right)^{\prime}=$ $\left(\phi_{p}\left(D_{0+}^{\alpha} u(0)\right)\right)^{\prime \prime}=\left(\phi_{p}\left(D_{0+}^{\alpha} u(0)\right)\right)^{\prime \prime \prime}=0$, we have

$$
c_{0}=c_{2}=c_{3}=0, \quad c_{1}=\int_{0}^{1} \frac{(\beta-1)(1-\tau)^{\beta-2}}{\Gamma(\beta)} y(\tau) d \tau .
$$

Therefore, the solution $u(t)$ of fractional differential equation boundary-value problems (18) and (19) satisfies

$$
\begin{aligned}
\phi_{p}\left(D_{0+}^{\alpha} u(t)\right)= & -\int_{0}^{t} \frac{(t-\tau)^{\beta-1}}{\Gamma(\beta)} y(\tau) d \tau \\
& +t \int_{0}^{1} \frac{(\beta-1)(1-\tau)^{\beta-2}}{\Gamma(\beta)} y(\tau) d \tau \\
= & \int_{0}^{1} H(t, \tau) y(\tau) d \tau .
\end{aligned}
$$

Consequently, $D_{0+}^{\alpha} u(t)=\phi_{q}\left(\int_{0}^{1} H(t, \tau) y(\tau) d \tau\right)$. Thus, fractional differential equation boundary-value problem (18) and (19) is equivalent to the problem

$$
\begin{gathered}
D_{0+}^{\alpha} u(t)=\phi_{q}\left(\int_{0}^{1} H(t, \tau) y(\tau) d \tau\right), \quad 0<t<1, \\
u(0)=\gamma u(h)+\lambda, \quad u^{\prime}(0)=\mu .
\end{gathered}
$$

Lemma 7 implies that the fractional differential equation boundary-value problems (18) and (19) have a unique solution,

$$
\begin{aligned}
u(t)= & \int_{0}^{t} \frac{(t-s)^{\alpha-1}}{\Gamma(\alpha)} \phi_{q}\left(\int_{0}^{1} H(s, \tau) y(\tau) d \tau\right) d s \\
& +\frac{\gamma}{1-\gamma} \int_{0}^{h} \frac{(h-s)^{\alpha-1}}{\Gamma(\alpha)} \phi_{q}\left(\int_{0}^{1} H(s, \tau) y(\tau) d \tau\right) d s \\
& +\frac{\lambda+\gamma \mu h}{1-\gamma}
\end{aligned}
$$

The proof is complete. 
Lemma 9 (see [15]). Let $1<\alpha \leqslant 2,3<\beta \leqslant 4$. The function $H(t, s)$ is continuous on $[0,1] \times[0,1]$ and satisfies

(1) $H(t, s) \geqslant 0, H(t, s) \leqslant H(1, s)$, for $t, s \in[0,1]$;

(2) $H(t, s) \geqslant t^{\beta-1} H(1, s)$, for $t, s \in(0,1)$.

Lemma 10 (Schauder fixed point theorem [30]). Let $(E, d)$ be a complete metric space, $U$ be a closed convex subset of $E$, and $A: U \rightarrow U$ be a mapping such that the set $\{A u: u \in U\}$ is relatively compact in $E$. Then, $A$ has at least one fixed point. tions.

To prove our main results, we use the following assump-

(H1) $0<\int_{0}^{1} H(1, \tau) a(\tau) d \tau<+\infty$;

(H2) there exist $0<\sigma<1$ and $c>0$ such that

$$
f(x) \leqslant \sigma L \phi_{p}(x), \quad \text { for } 0 \leqslant x \leqslant c,
$$

where $L$ satisfies

$$
0<L \leqslant\left[\phi_{p}\left(\frac{1+\gamma\left(h^{\alpha}-1\right)}{\Gamma(\alpha+1)(1-\gamma)}\right) \int_{0}^{1} H(1, \tau) a(\tau) d \tau\right]^{-1} ;
$$

(H3) there exist $d>0$ such that

$$
f(x) \leqslant M \phi_{p}(x), \quad \text { for } d<x<+\infty,
$$

where $M$ satisfies

$0<M$

$$
<\left[\phi_{p}\left(\frac{1+\gamma\left(h^{\alpha}-1\right)}{\Gamma(\alpha+1)(1-\gamma)} 2^{q-1}\right) \int_{0}^{1} H(1, \tau) a(\tau) d \tau\right]^{-1} ;
$$

(H4) there exist $0<\delta<1$ and $e>0$ such that

$$
f(x) \geqslant N \phi_{p}(x), \quad \text { for } e<x<+\infty,
$$

where $N$ satisfies

$$
\begin{gathered}
N>\left[\phi_{p}\left(c_{\delta} \int_{0}^{1} \frac{(1-s)^{\alpha-2}}{\Gamma(\alpha)} \phi_{q}\left(s^{\beta-1}\right) d s\right)\right. \\
\left.\times \int_{\delta}^{1} H(1, \tau) a(\tau) d \tau\right]^{-1} ;
\end{gathered}
$$

with

$$
c_{\delta}=\int_{0}^{\delta} \alpha(1-s)^{\alpha-2} \phi_{q}\left(s^{\beta-1}\right) d s \in(0,1) ;
$$

(H5) $f(x)$ is nondecreasing in $x$;

(H6) there exist $0 \leqslant \theta<1$ such that

$f(k x) \geqslant\left(\phi_{p}(k)\right)^{\theta} f(x), \quad$ for any $0<k<1,0<x<+\infty$.
Remark 11. Let

$$
f_{0}=\lim _{x \rightarrow 0^{+}} \frac{f(x)}{\phi_{p}(x)}, \quad f_{\infty}=\lim _{x \rightarrow+\infty} \frac{f(x)}{\phi_{p}(x)} .
$$

Then, (H2) holds if $f_{0}=0$, (H3) holds if $f_{\infty}=0$, and (H4) holds if $f_{\infty}=+\infty$.

\section{Existence}

Theorem 12. Assume that (H1), (H2) hold. Then, the fractional differential equation boundary-value problem (3) has at least one positive solution for $0<\lambda+\gamma \mu \leq(1-\gamma)\left(1-\phi_{q}(\sigma)\right) c$.

Proof. Let $c>0$ be given in (H2). Define

$$
K_{1}=\{u \in C[0,1]: 0 \leqslant u(t) \leqslant c \text { on }[0,1]\}
$$

and an operator $T_{\lambda}: K_{1} \rightarrow C[0,1]$ by

$$
\begin{aligned}
T_{\lambda} u(t)= & \int_{0}^{t} \frac{(t-s)^{\alpha-1}}{\Gamma(\alpha)} \phi_{q}\left(\int_{0}^{1} H(s, \tau) a(\tau) f(u(\tau)) d \tau\right) \\
& +\frac{\gamma}{1-\gamma} \int_{0}^{h} \frac{(h-s)^{\alpha-1}}{\Gamma(\alpha)} \phi_{q} \\
& \quad \times\left(\int_{0}^{1} H(s, \tau) a(\tau) f(u(\tau)) d \tau\right) d s \\
& +\frac{\lambda+\gamma \mu h}{1-\gamma} .
\end{aligned}
$$

Then, $K_{1}$ is a closed convex set. From Lemma $8, u$ is a solution of fractional differential equation boundary-value problem (3) if and only if $u$ is a fixed point of $T_{\lambda}$. Moreover, a standard argument can be used to show that $T_{\lambda}$ is compact.

For any $u \in K_{1}$, from (28) and (29), we obtain

$$
\begin{gathered}
f(u(t)) \leqslant \sigma L \phi_{p}(u(t)) \leqslant \sigma L \phi_{p}(c), \quad \text { on }[0,1], \\
\frac{1+\gamma\left(h^{\alpha}-1\right)}{\Gamma(\alpha+1)(1-\gamma)} \phi_{q}(L) \phi_{q}\left(\int_{0}^{1} H(1, \tau) a(\tau) d \tau\right) \leqslant 1 .
\end{gathered}
$$

Let $0<\lambda+\gamma \mu \leqslant(1-\gamma)\left(1-\phi_{q}(\sigma)\right) c$. Then, from Lemma 6 and (38), it follows that

$$
0 \leqslant T_{\lambda} u(t)
$$

$$
\begin{aligned}
\leqslant & \int_{0}^{t} \frac{(t-s)^{\alpha-1}}{\Gamma(\alpha)} \phi_{q}\left(\int_{0}^{1} H(1, \tau) a(\tau) f(u(\tau)) d \tau\right) d s \\
& +\frac{\gamma}{1-\gamma}
\end{aligned}
$$

$$
\times \int_{0}^{h} \frac{(h-s)^{\alpha-1}}{\Gamma(\alpha)} \phi_{q}\left(\int_{0}^{1} H(1, \tau) a(\tau) f(u(\tau)) d \tau\right) d s
$$$$
+\frac{\lambda+\gamma \mu h}{1-\gamma}
$$ 


$$
\begin{aligned}
\leqslant & \frac{1}{\Gamma(\alpha+1)} \phi_{q}\left(\int_{0}^{1} H(1, \tau) a(\tau) f(u(\tau)) d \tau\right) \\
& +\frac{\gamma h^{\alpha}}{\Gamma(\alpha+1)(1-\gamma)} \phi_{q} \\
& \times\left(\int_{0}^{1} H(1, \tau) a(\tau) f(u(\tau)) d \tau\right)+\left(1-\phi_{q}(\sigma)\right) c \\
= & \frac{1+\gamma h^{\alpha}-\gamma}{\Gamma(\alpha+1)(1-\gamma)} \phi_{q}\left(\int_{0}^{1} H(1, \tau) a(\tau) f(u(\tau)) d \tau\right) \\
& +\left(1-\phi_{q}(\sigma)\right) c \\
\leqslant & \frac{1+\gamma\left(h^{\alpha}-1\right)}{\Gamma(\alpha+1)(1-\gamma)} \phi_{q}(L) \phi_{q}\left(\int_{0}^{1} H(1, \tau) a(\tau) d \tau\right) \phi_{q}(\sigma) c \\
& +\left(1-\phi_{q}(\sigma)\right) c \\
\leqslant & \phi_{q}(\sigma) c+\left(1-\phi_{q}(\sigma)\right) c=c, \quad t \in[0,1] .
\end{aligned}
$$

Thus, $T_{\lambda}\left(K_{1}\right) \subseteq K_{1}$, by Schauder fixed point theorem, $T_{\lambda}$ has a fixed point $u \in K_{1}$; that is, the fractional differential equation boundary-value problem (3) has at least one positive solution. The proof is complete.

Corollary 13. Assume that (H1) holds and $f_{0}=0$. Then, the fractional differential equation boundary-value problem (3) has at least one positive solution for sufficiently small $\lambda>0$.

Theorem 14. Assume that (H1), (H3) hold. Then, the fractional differential equation boundary-value problem (3) has at least one positive solution for all $\lambda>0$.

Proof. Let $\lambda>0$ be fixed and $d>0$ be given in (H3). Define $D=\max _{0 \leqslant x \leqslant d} f(x)$. Then

$$
f(x) \leqslant D, \quad \text { for } 0 \leqslant x \leqslant d .
$$

From (31), we have

$$
\frac{1+\gamma\left(h^{\alpha}-1\right)}{\Gamma(\alpha+1)(1-\gamma)} 2^{q-1} \phi_{q}(M) \phi_{q}\left(\int_{0}^{1} H(1, \tau) a(\tau) d \tau\right)<1 .
$$

Thus, there exists $d^{*}>d$ large enough so that

$$
\begin{aligned}
& \frac{1+\gamma\left(h^{\alpha}-1\right)}{\Gamma(\alpha+1)(1-\gamma)} 2^{q-1}\left(\phi_{q}(D)+\phi_{q}(M) d^{*}\right) \phi_{q} \\
& \quad \times\left(\int_{0}^{1} H(1, \tau) a(\tau) d \tau\right)+\frac{\lambda+\gamma \mu h}{1-\gamma} \leqslant d^{*} .
\end{aligned}
$$

Let

$$
K_{2}=\left\{u \in C[0,1]: 0 \leqslant u(t) \leqslant d^{*} \text { on }[0,1]\right\} .
$$

For $u \in K_{2}$, define

$$
\begin{gathered}
I_{1}^{u}=\{t \in[0,1]: 0 \leqslant u(t) \leqslant d\}, \\
I_{2}^{u}=\left\{t \in[0,1]: d<u(t) \leqslant d^{*}\right\} .
\end{gathered}
$$

Then, $I_{1}^{u} \cup I_{2}^{u}=[0,1], I_{1}^{u} \cap I_{2}^{u}=\emptyset$, and in view of (30), we have

$$
f(u(t)) \leqslant M \phi_{p}(u(t)) \leqslant M \phi_{p}\left(d^{*}\right), \quad \text { for } t \in I_{2}^{u} .
$$

Let the compact operator $T_{\lambda}$ be defined by (38). Then, from Lemma 6, (30), and (41), we have

$$
\begin{aligned}
& 0 \leqslant T_{\lambda} u(t) \\
& \leqslant \int_{0}^{t} \frac{(t-s)^{\alpha-1}}{\Gamma(\alpha)} \phi_{q}\left(\int_{0}^{1} H(1, \tau) a(\tau) f(u(\tau)) d \tau\right) d s \\
& +\frac{\gamma}{1-\gamma} \\
& \times \int_{0}^{h} \frac{(h-s)^{\alpha-1}}{\Gamma(\alpha)} \phi_{q}\left(\int_{0}^{1} H(1, \tau) a(\tau) f(u(\tau)) d \tau\right) d s \\
& +\frac{\lambda+\gamma \mu h}{1-\gamma} \\
& \leqslant \frac{1}{\Gamma(\alpha+1)} \phi_{q}\left(\int_{0}^{1} H(1, \tau) a(\tau) f(u(\tau)) d \tau\right) \\
& +\frac{\gamma h^{\alpha}}{\Gamma(\alpha+1)(1-\gamma)} \phi_{q}\left(\int_{0}^{1} H(1, \tau) a(\tau) f(u(\tau)) d \tau\right) \\
& +\frac{\lambda+\gamma \mu h}{1-\gamma} \\
& =\frac{1+\gamma\left(h^{\alpha}-1\right)}{\Gamma(\alpha+1)(1-\gamma)} \phi_{q} \\
& \times\left(\int_{I_{1}^{u}} H(1, \tau) a(\tau) f(u(\tau)) d \tau\right. \\
& \left.+\int_{I_{2}^{u}} H(1, \tau) a(\tau) f(u(\tau)) d \tau\right) \\
& +\frac{\lambda+\gamma \mu h}{1-\gamma} \\
& \leqslant \frac{1+\gamma\left(h^{\alpha}-1\right)}{\Gamma(\alpha+1)(1-\gamma)} \phi_{q} \\
& \times\left(D \int_{I_{1}^{u}} H(1, \tau) a(\tau) d \tau\right. \\
& \left.+M \phi_{p}\left(d^{*}\right) \int_{I_{2}^{u}} H(1, \tau) a(\tau) d \tau\right) \\
& +\frac{\lambda+\gamma \mu h}{1-\gamma} \\
& \leqslant \frac{1+\gamma\left(h^{\alpha}-1\right)}{\Gamma(\alpha+1)(1-\gamma)} \phi_{q}\left(D+M \phi_{p}\left(d^{*}\right)\right) \phi_{q} \\
& \times\left(\int_{0}^{1} H(1, \tau) a(\tau) d \tau\right)+\frac{\lambda+\gamma \mu h}{1-\gamma} .
\end{aligned}
$$


From (43) and the inequality $(a+b)^{r} \leqslant 2^{r}\left(a^{r}+b^{r}\right)$ for any $a, b, r>0$ (see, e.g., [31]), we obtain

$$
\begin{aligned}
0 \leqslant & T_{\lambda} u(t) \\
\leqslant & \frac{1+\gamma\left(h^{\alpha}-1\right)}{\Gamma(\alpha+1)(1-\gamma)} 2^{q-1}\left(\phi_{q}(D)+\phi_{q}(M) d^{*}\right) \phi_{q} \\
& \times\left(\int_{0}^{1} H(1, \tau) a(\tau) d \tau\right)+\frac{\lambda+\gamma \mu h}{1-\gamma} \leqslant d^{*} .
\end{aligned}
$$

Thus, $T_{\lambda}: K_{2} \rightarrow K_{2}$. Consequently, by Schauder fixed point theorem, $T_{\lambda}$ has a fixed point $u \in K_{2}$, that is, the fractional differential equation boundary-value problem (3) has at least one positive solution. The proof is complete.

Corollary 15. Assume that (H1) holds and $f_{\infty}=0$. Then, the fractional differential equation boundary-value problem (3) has at least one positive solution for all $\lambda>0$.

\section{Uniqueness}

Definition 16 (see [32]). A cone $P$ in a real Banach space $X$ is called solid if its interior $P^{o}$ is not empty.

Definition 17 (see [32]). Let $P$ be a solid cone in a real Banach space $X, T: P^{o} \rightarrow P^{o}$ be an operator, and $0 \leqslant \theta<1$. Then, $T$ is called a $\theta$-concave operator if

$$
T(k u) \geqslant k^{\theta} T u \quad \text { for any } 0<k<1, u \in P^{o} .
$$

Lemma 18 (see [32, Theorem 2.2.6]). Assume that $P$ is a normal solid cone in a real Banach space $X, 0 \leqslant \theta<1$, and $T: P^{o} \rightarrow P^{o}$ is a $\theta$-concave increasing operator. Then, $T$ has only one fixed point in $P^{o}$.

Theorem 19. Assume that (H1), (H5), (H6) hold. Then, the fractional differential equation boundary-value problem (3) has a unique positive solution for any $\lambda>0$.

Proof. Define $P=\{u \in C[0,1]: u(t) \geqslant 0$ on $[0,1]\}$. Then, $P$ is a normal solid cone in $C[0,1]$ with

$$
P^{o}=\{u \in C[0,1]: u(t)>0 \text { on }[0,1]\} .
$$

For any fixed $\lambda>0$, let $T_{\lambda}: P \rightarrow C[0,1]$ be defined by (38). Define $T: P \rightarrow C[0,1]$ by

$$
\begin{aligned}
& T u(t)= \int_{0}^{t} \frac{(t-s)^{\alpha-1}}{\Gamma(\alpha)} \phi_{q}\left(\int_{0}^{1} H(s, \tau) a(\tau) f(u(\tau)) d \tau\right) d s \\
&+\frac{\gamma}{1-\gamma} \int_{0}^{h} \frac{(h-s)^{\alpha-1}}{\Gamma(\alpha)} \phi_{q} \\
& \quad \times\left(\int_{0}^{1} H(s, \tau) a(\tau) f(u(\tau)) d \tau\right) d s .
\end{aligned}
$$

Then, from (H5), we have $T$ increasing in $u \in P^{o}$ and

$$
T_{\lambda} u(t)=T u(t)+\frac{\lambda+\gamma \mu h}{1-\gamma} .
$$

Clearly, $T_{\lambda}: P^{o} \rightarrow P^{o}$. Next, we prove that $T_{\lambda}$ is a $\theta$-concave increasing operator. In fact, for $u_{1}, u_{2} \in P$ with $u_{1}(t) \geqslant u_{2}(t)$ on $[0,1]$, we obtain

$$
T_{\lambda} u_{1}(t) \geqslant T u_{2}(t)+\frac{\lambda+\gamma \mu h}{1-\gamma}=T_{\lambda} u_{2}(t)
$$

that is, $T_{\lambda}$ is increasing. Moreover, (H6) implies

$$
\begin{aligned}
T_{\lambda}(k u)(t) \geqslant & k^{\theta} \int_{0}^{t} \frac{(t-s)^{\alpha-1}}{\Gamma(\alpha)} \\
& \quad \times \phi_{q}\left(\int_{0}^{1} H(s, \tau) a(\tau) f(u(\tau)) d \tau\right) d s \\
& +k^{\theta} \frac{\gamma}{1-\gamma} \\
& \times \int_{0}^{h} \frac{(h-s)^{\alpha-1}}{\Gamma(\alpha)} \\
& +\frac{\lambda+\gamma \mu h}{1-\gamma} \\
= & k^{\theta} T^{\theta} u(t)+\frac{\lambda+\gamma \mu h}{1-\gamma} \\
& k^{\theta}\left(T u(t)+\frac{\lambda+\gamma \mu h}{1-\gamma}\right)=k^{\theta} T_{\lambda} u(t),
\end{aligned}
$$

that is, $T_{\lambda}$ is $\theta$-concave. By Lemma $8, T_{\lambda}$ has a unique fixed point $u_{\lambda}$ in $P^{o}$, that is, the fractional differential equation boundary-value problem (3) has a unique positive solution. The proof is complete.

\section{Nonexistence}

In this section, we let the Banach space $C[0,1]$ be endowed with the norm $\|u\|=\max _{0 \leqslant t \leqslant 1}|u(t)|$.

Lemma 20. Assume (H1) holds and let $0<\delta<1$ be given in (H4). Then, the unique solution $u(t)$ of fractional differential equation boundary-value problem (18) and (19) satisfies

$$
u(t) \geqslant c_{\delta}\|u\| \quad \text { for } \delta \leqslant t \leqslant 1
$$

where $c_{\delta}$ is defined by (34). 
Proof. In view of Lemma 9 and (19), we have

$$
\begin{aligned}
u(t) \leqslant & \int_{0}^{t} \frac{(t-s)^{\alpha-1}}{\Gamma(\alpha)} \phi_{q}\left(\int_{0}^{1} H(1, \tau) y(\tau) d \tau\right) d s \\
& +\frac{\gamma}{1-\gamma} \\
& \times \int_{0}^{h} \frac{(h-s)^{\alpha-1}}{\Gamma(\alpha)} \phi_{q}\left(\int_{0}^{1} H(s, \tau) y(\tau) d \tau\right) d s \\
& +\frac{\lambda+\gamma \mu h}{1-\gamma} \\
\leqslant & \frac{1}{\Gamma(\alpha+1)} \phi_{q}\left(\int_{0}^{1} H(1, \tau) y(\tau) d \tau\right) \\
& +\frac{\gamma}{1-\gamma} \\
& \times \int_{0}^{h} \frac{(h-s)^{\alpha-1}}{\Gamma(\alpha)} \phi_{q}\left(\int_{0}^{1} H(s, \tau) y(\tau) d \tau\right) d s \\
& +\frac{\lambda+\gamma \mu h}{1-\gamma}
\end{aligned}
$$

for $t \in[0,1]$, and

$$
\begin{aligned}
u(t) \geqslant & \int_{0}^{t} \frac{(t-s)^{\alpha-1}}{\Gamma(\alpha)} \phi_{q}\left(\int_{0}^{1} s^{\beta-1} H(1, \tau) y(\tau) d \tau\right) d s \\
& +\frac{\gamma}{1-\gamma} \\
& \times \int_{0}^{h} \frac{(h-s)^{\alpha-1}}{\Gamma(\alpha)} \phi_{q}\left(\int_{0}^{1} H(s, \tau) y(\tau) d \tau\right) d s \\
& +\frac{\lambda+\gamma \mu h}{1-\gamma} \\
= & \int_{0}^{t} \alpha(t-s)^{\alpha-2} \phi_{q}\left(s^{\beta-1}\right) d s \frac{1}{\Gamma(\alpha+1)} \phi_{q} \\
& \times\left(\int_{0}^{1} H(1, \tau) y(\tau) d \tau\right) \\
& +\frac{\gamma}{1-\gamma} \int_{0}^{h} \frac{(h-s)^{\alpha-1}}{\Gamma(\alpha)} \phi_{q} \\
& +\frac{\gamma}{1-\gamma} \times\left(\int_{0}^{1} H(s, \tau) y(\tau) d \tau\right) d s+\frac{\lambda+\gamma \mu h}{1-\gamma} \\
& \frac{1}{\Gamma(\alpha+1)} \phi_{q}\left(\int_{0}^{1} H(1, \tau) y(\tau) d \tau\right)
\end{aligned}
$$

$$
\begin{gathered}
\times \int_{0}^{h} \frac{(h-s)^{\alpha-1}}{\Gamma(\alpha)} \phi_{q}\left(\int_{0}^{1} H(s, \tau) y(\tau) d \tau\right) d s \\
+\frac{\lambda+\gamma \mu h}{1-\gamma} \\
\geqslant c_{\delta}\left[\frac{1}{\Gamma(\alpha+1)} \phi_{q}\left(\int_{0}^{1} H(1, \tau) y(\tau) d \tau\right)\right. \\
+\frac{\gamma}{1-\gamma} \int_{0}^{h} \frac{(h-s)^{\alpha-1}}{\Gamma(\alpha)} \\
\left.+\frac{\lambda+\gamma \mu h}{1-\gamma}\right]
\end{gathered}
$$

for $t \in[\delta, 1]$. Therefore, $u(t) \geqslant c_{\delta}\|u\|$ for $\delta \leqslant t \leqslant 1$. The proof is complete.

Theorem 21. Assume that (H1), (H4) hold. Then, the fractional differential equation boundary-value problem (3) has no positive solution for $\lambda+\gamma \mu h>(1-\gamma) e$.

Proof. Assume, to the contrary, the fractional differential equation boundary-value problem (3) has a positive solution $u(t)$ for $\lambda+\gamma \mu h>(1-\gamma) e$. Then, by Lemma 8 , we have

$$
\begin{aligned}
u(t)= & \int_{0}^{t} \frac{(t-s)^{\alpha-1}}{\Gamma(\alpha)} \phi_{q} \\
& \times\left(\int_{0}^{1} H(s, \tau) a(\tau) f(u(\tau)) d \tau\right) d s \\
& +\frac{\gamma}{1-\gamma} \\
& \times \int_{0}^{h} \frac{(h-s)^{\alpha-1}}{\Gamma(\alpha)} \phi_{q}\left(\int_{0}^{1} H(s, \tau) a(\tau) f(u(\tau)) d \tau\right) d s \\
& +\frac{\lambda+\gamma \mu h}{1-\gamma} .
\end{aligned}
$$

Therefore, $u(t)>e$ on $[0,1]$. In view of (32) and (33), we obtain

$$
\begin{gathered}
f(u(t)) \geqslant N \phi_{p}(u(t)) \quad \text { on }[0,1], \\
c_{\delta} \phi_{q}(N) \phi_{q}\left(\int_{\delta}^{1} H(1, \tau) a(\tau) d \tau\right) \\
\times \int_{0}^{1} \frac{(1-s)^{\alpha-1}}{\Gamma(\alpha)} \phi_{q}\left(s^{\beta-1}\right) d s>1 .
\end{gathered}
$$

Then, by Lemmas 6 and 9, we obtain

$$
\begin{aligned}
\|u\| \geq & u(1) \\
> & \int_{0}^{1} \frac{(1-s)^{\alpha-1}}{\Gamma(\alpha)} \phi_{q} \\
& \quad \times\left(\int_{0}^{1} H(s, \tau) a(\tau) f(u(\tau)) d \tau\right) d s
\end{aligned}
$$




$$
\begin{aligned}
\geqslant & \int_{0}^{1} \frac{(1-s)^{\alpha-1}}{\Gamma(\alpha)} \phi_{q}\left(s^{\beta-1}\right) d s \phi_{q} \\
& \times\left(\int_{0}^{1} H(1, \tau) a(\tau) f(u(\tau)) d \tau\right) \\
\geqslant & \int_{0}^{1} \frac{(1-s)^{\alpha-1}}{\Gamma(\alpha)} \phi_{q}\left(s^{\beta-1}\right) d s \phi_{q}(N) \phi_{q} \\
& \times\left(\int_{\delta}^{1} H(1, \tau) a(\tau) \phi_{p}(u(\tau)) d \tau\right) \\
\geqslant & \|u\| c_{\delta} \int_{0}^{1} \frac{(1-s)^{\alpha-1}}{\Gamma(\alpha)} \phi_{q}\left(s^{\beta-1}\right) d s \phi_{q}(N) \phi_{q} \\
& \times\left(\int_{\delta}^{1} H(1, \tau) a(\tau) d \tau\right)
\end{aligned}
$$$$
>\|u\| \text {. }
$$

This contradiction completes the proof.

Corollary 22. Assume that (H1) holds and $f_{\infty}=+\infty$. Then, the fractional differential equation boundary-value problem (3) has no positive solution for sufficiently large $\lambda>0$.

\section{Conclusion: Identities on$$
\text { the Special Polynomials whereby }
$$$$
\text { Caputo Fractional Derivative }
$$

In this final part, we will focus on the new interesting identities related to special polynomials by means of Caputo fractional derivative.

As well known, the Bernoulli polynomials may be defined to be

$$
F(t, z)=\frac{z}{e^{z}-1} e^{t z}=e^{B z}=\sum_{n=0}^{\infty} B_{n}(t) \frac{z^{n}}{n !},
$$

where usual convention about replacing $B^{n}$ by $B_{n}$ in is used. Also, we note that the Bernoulli polynomials is analytic on the region $D=\{z \in \mathbb{C}|| z \mid<2 \pi\}$ (see [33]).

Let $d / d t$ be familiar normal derivative, then we can obtain the following identity

$$
\frac{d}{d t} t^{n}=n t^{n-1}
$$

Differentiating in both sides of (61), we have

$$
\frac{d}{d t} B_{n}(t)=n B_{n-1}(t)
$$

(see [33]).

When $t=0$ in (61), we have $B_{n}(0):=B_{n}$ are called Bernoulli numbers, which can be generated by

$$
F(z)=\frac{z}{e^{z}-1}=\sum_{n=0}^{\infty} B_{n} \frac{z^{n}}{n !} .
$$

By (61) and (64), we have the following functional equation:

$$
F(t, z)=e^{t z} F(z)
$$

and this equation yields to

$$
\begin{aligned}
B_{m}(t) & =\sum_{k=0}^{m}\left(\begin{array}{c}
m \\
k
\end{array}\right) t^{m-k} B_{k} \\
& =\sum_{k=0}^{m}\left(\begin{array}{c}
m \\
k
\end{array}\right) t^{k} B_{m-k}
\end{aligned}
$$

(see [33]).

Let us now take $y(t)=B_{m}(t)$ in Definition 2 leads to

$$
\begin{aligned}
D_{0+}^{\alpha} B_{m}(t)= & \frac{1}{\Gamma(n-\alpha)} \int_{0}^{t} \frac{\left.\left(d^{n} / d t^{n}\right) B_{m}(t)\right|_{t=s}}{(t-s)^{\alpha-n+1}} d s \\
= & m(m-1) \cdots(m-n+1) \\
& \times \sum_{k=0}^{m-n}\left(\begin{array}{c}
m-n \\
k
\end{array}\right) B_{m-n-k} \\
& \times\left[\frac{1}{\Gamma(n-\alpha)} \int_{0}^{t} \frac{s^{k}}{(t-s)^{\alpha-n+1}} d s\right] \\
= & \frac{\Gamma(m+1)}{\Gamma(m-n+1)} \sum_{k=0}^{m-n} \frac{k !\left(\begin{array}{c}
m-n \\
k
\end{array}\right) B_{m-n-k}}{\Gamma(n+k-\alpha+1)} t^{k-\alpha+n} .
\end{aligned}
$$

Therefore, we procure the following theorem.

Theorem 23. The following identity holds true:

$$
\begin{aligned}
D_{0+}^{\alpha} B_{m}(t)= & \frac{\Gamma(m+1)}{\Gamma(m-n+1)} \\
& \times \sum_{k=0}^{m-n} \frac{k !\left(\begin{array}{c}
m-n \\
k
\end{array}\right) B_{m-n-k}}{\Gamma(n+k-\alpha+1)} t^{k-\alpha+n} .
\end{aligned}
$$

In [34], the Bernoulli polynomials of higher order are defined by

$$
\underbrace{\frac{z}{e^{z}-1} \frac{z}{e^{z}-1} \cdots \frac{z}{e^{z}-1}}_{l \text {-times }} e^{t z}=\sum_{m=0}^{\infty} B_{m}^{(l)}(t) \frac{z^{n}}{n !},
$$

we note that $B_{m}^{(l)}(t)$ is analytic on $D$. It follows from (69), we have

$$
\begin{gathered}
\frac{d}{d t} B_{m}^{(l)}(t)=m B_{m-1}^{(l)}(t) \\
\frac{d^{n}}{d t^{n}} B_{m}^{(l)}(t)=\frac{\Gamma(m+1)}{\Gamma(m-n+1)} B_{m-n}^{(l)}(t)
\end{gathered}
$$

(see [34]).

Substituting $x=0$ into $(69), B_{m}^{(l)}(0):=B_{m}^{(l)}$ are called Bernoulli polynomials of higher order. 
Owing to (69) and (70), we readily see that

$$
\begin{aligned}
D_{0+}^{\alpha} B_{m}^{(l)}(t)= & \frac{1}{\Gamma(n-\alpha)} \int_{0}^{t} \frac{\left.\left(d^{n} / d t^{n}\right) B_{m}^{(l)}(t)\right|_{t=s}}{(t-s)^{\alpha-n+1}} d s \\
= & m(m-1) \cdots(m-n+1) \\
& \times \sum_{k=0}^{m-n}\left(\begin{array}{c}
m-n \\
k
\end{array}\right) B_{m-n-k}^{(l)} \\
& \times\left[\frac{1}{\Gamma(n-\alpha)} \int_{0}^{t} \frac{s^{k}}{\left.(t-s)^{\alpha-n+1} d s\right]}\right] \\
= & \frac{\Gamma(m+1)}{\Gamma(m-n+1)} \sum_{k=0}^{m-n} \frac{k !\left(\begin{array}{c}
m-n \\
k
\end{array}\right) B_{m-n-k}^{(l)}}{\Gamma(n+k-\alpha+1)} t^{k-\alpha+n} \\
= & \frac{\Gamma(m+1)}{\Gamma(m-n+1)} \sum_{k=0}^{m-n} \frac{k !\left(\begin{array}{c}
m-n \\
k
\end{array}\right)}{\Gamma(n+k-\alpha+1)} t^{k-\alpha+n} \\
& \left.\times\left(\begin{array}{c}
\sum_{s_{1}+s_{2}+\cdots+s_{l}=m-n-k} s_{s_{l} \geq 0}, s_{2}, \ldots, s_{l}
\end{array}\right)\left(\prod_{j=1}^{l} B_{s_{j}}\right)\right) .
\end{aligned}
$$

Therefore, we can state the following theorem.

Theorem 24. The following identity holds true:

$$
\begin{aligned}
D_{0+}^{\alpha} B_{m}^{(l)}(t)= & \frac{\Gamma(m+1)}{\Gamma(m-n+1)} \sum_{k=0}^{m-n} \frac{k !\left(\begin{array}{c}
m-n \\
k
\end{array}\right)}{\Gamma(n+k-\alpha+1)} t^{k-\alpha+n} \\
& \left.\times\left(\begin{array}{c}
\sum_{\substack{s_{1}+s_{2}+\cdots+s_{l}=m-n-k \\
s_{l} \geq 0}}\left(\begin{array}{c}
m-n-k \\
s_{1}, s_{2}, \ldots, s_{l}
\end{array}\right)\left(\prod_{j=1}^{l} B_{s_{j}}\right.
\end{array}\right)\right),
\end{aligned}
$$

in which $B_{s_{j}}$ and $\left(\begin{array}{c}m-n-k \\ s_{1}, s_{2}, \ldots, s_{l}\end{array}\right)$ are Bernoulli numbers and multibinomial coefficients.

In the region $T=\{z \in \mathbb{C}|| z \mid<\pi\}$, the Euler polynomials and the Euler polynomials of higher order are given, respectively, with the help of the following generating functions:

$$
\begin{gathered}
\frac{2}{e^{z}+1} e^{t z}=\sum_{m=0}^{\infty} E_{m}(t) \frac{z^{m}}{m !} \\
\underbrace{\frac{2}{e^{z}+1} \frac{2}{e^{z}+1} \cdots \frac{2}{e^{z}+1} e^{t z}}_{l \text {-times }} \\
=\sum_{m=0}^{\infty}\left(\sum_{s_{1}+s_{2}+\cdots+s_{l}=m}\left(\begin{array}{c}
m \\
s_{1}, s_{2}, \ldots, s_{l}
\end{array}\right)\left(\prod_{j=1}^{l-1} E_{s_{j}}\right) t^{s_{l}}\right) \frac{z^{m}}{m !} \\
=\sum_{m=0}^{\infty} E_{m}^{(l)}(t) \frac{z^{n}}{n !},
\end{gathered}
$$

where $E_{s_{j}}$ are Euler numbers in (see [33-36]). From the last equation, we discover the following:

$$
\begin{aligned}
& \frac{d}{d t} E_{m}(t)=m E_{m-1}(t), \\
& \frac{d}{d t} E_{m}^{(l)}(t)=m E_{m-1}^{(l)}(t)
\end{aligned}
$$

(see [35]).

Obviously, we have that

$$
E_{m}^{(1)}(t):=E_{m}(t) .
$$

Taking $y(t)=E_{m}^{(l)}(t)$ in Definition 2, by (73) and (75), we compute

$$
\begin{aligned}
& D_{0+}^{\alpha} E_{m}^{(l)}(t)=\frac{1}{\Gamma(n-\alpha)} \int_{0}^{t} \frac{\left.\left(d^{n} / d t^{n}\right) E_{m}^{(l)}(t)\right|_{t=s}}{(t-s)^{\alpha-n+1}} d s \\
& =m(m-1) \cdots(m-n+1) \\
& \times \sum_{k=0}^{m-n}\left(\begin{array}{c}
m-n \\
k
\end{array}\right) E_{m-n-k}^{(l)} \\
& \times\left[\frac{1}{\Gamma(n-\alpha)} \int_{0}^{t} \frac{s^{k}}{(t-s)^{\alpha-n+1}} d s\right] \\
& =\frac{\Gamma(m+1)}{\Gamma(m-n+1)} \sum_{k=0}^{m-n} \frac{k !\left(\begin{array}{c}
m-n \\
k
\end{array}\right) E_{m-n-k}^{(l)}}{\Gamma(n+k-\alpha+1)} t^{k-\alpha+n} \\
& =\frac{\Gamma(m+1)}{\Gamma(m-n+1)} \sum_{k=0}^{m-n} \frac{k !\left(\begin{array}{c}
m-n \\
k
\end{array}\right)}{\Gamma(n+k-\alpha+1)} t^{k-\alpha+n} \\
& \times\left(\sum_{\substack{s_{1}+s_{2}+\cdots+s_{l}=m-n-k \\
s_{l} \geq 0}}\left(\begin{array}{c}
m-n-k \\
s_{1}, s_{2}, \ldots, s_{l}
\end{array}\right)\left(\prod_{j=1}^{l} E_{s_{j}}\right)\right) .
\end{aligned}
$$

Therefore, we obtain the following theorem.

Theorem 25. The following identity

$$
\begin{aligned}
D_{0+}^{\alpha} E_{m}^{(l)}(t) & \\
= & \frac{\Gamma(m+1)}{\Gamma(m-n+1)} \sum_{k=0}^{m-n} \frac{k !\left(\begin{array}{c}
m-n \\
k
\end{array}\right)}{\Gamma(n+k-\alpha+1)} \\
& \left.\times\left(\begin{array}{c}
\sum_{\substack{1 \\
s_{1}+s_{2}+\cdots+s_{l}=m-n-k \\
s_{l} \geq 0}}\left(\begin{array}{c}
m-n-k \\
s_{1}, s_{2}, \ldots, s_{l}
\end{array}\right)\left(\prod_{j=1}^{l} E_{s_{j}}\right.
\end{array}\right)\right) t^{k-\alpha+n}
\end{aligned}
$$

is true. Obviously, we have that

$$
D_{0+}^{\alpha} E_{m}(t)=\frac{\Gamma(m+1)}{\Gamma(m-n+1)} \sum_{k=0}^{m-n} \frac{k !\left(\begin{array}{c}
m-n \\
k
\end{array}\right) E_{m-n-k}}{\Gamma(n+k-\alpha+1)} t^{k-\alpha+n}
$$


In the region $T=\{z \in \mathbb{C}|| z \mid<\pi\}$, Genocchi polynomials, $G_{m}(x)$, and Genocchi polynomials of higher order, $G_{m}^{(l)}(x)$, are defined as an extension of Genocchi numbers $G_{m}$ defined in $[33,37,38]$, respectively,

$$
\begin{gathered}
\frac{2 z}{e^{z}+1} e^{t z}=\sum_{m=0}^{\infty} G_{m}(t) \frac{z^{m}}{m !}, \\
\underbrace{\frac{2 z}{e^{z}+1} \frac{2 z}{e^{z}+1} \cdots \frac{2 z}{e^{z}+1} e^{t z}}_{l \text {-times }}=\sum_{m=0}^{\infty} G_{m}^{(l)}(t) \frac{z^{n}}{n !} .
\end{gathered}
$$

In this final section, by the similar method, we arrive at the following theorem.

Theorem 26. The following identity

$$
\begin{aligned}
D_{0+}^{\alpha} G_{m}^{(l)}(t) & \frac{\Gamma(m+1)}{\Gamma(m-n+1)} \sum_{k=0}^{m-n} \frac{k !\left(\begin{array}{c}
m-n \\
k
\end{array}\right)}{\Gamma(n+k-\alpha+1)} \\
& \left.\times\left(\begin{array}{c}
\sum_{\substack{s_{1}+s_{2}+\cdots+s_{l}=m-n-k \\
s_{l} \geq 0}}\left(\begin{array}{c}
m-n-k \\
s_{1}, s_{2}, \ldots, s_{l}
\end{array}\right)\left(\prod_{j=1}^{l} G_{s_{j}}\right.
\end{array}\right)\right) t^{k-\alpha+n}
\end{aligned}
$$

is true. Obviously, we have that,

$$
D_{0+}^{\alpha} G_{m}(t)=\frac{\Gamma(m+1)}{\Gamma(m-n+1)} \sum_{k=0}^{m-n} \frac{k !\left(\begin{array}{c}
m-n \\
k
\end{array}\right) G_{m-n-k}}{\Gamma(n+k-\alpha+1)} t^{k-\alpha+n}
$$

\section{References}

[1] O. P. Agrawal, "Formulation of Euler-Lagrange equations for fractional variational problems," Journal of Mathematical Analysis and Applications, vol. 272, no. 1, pp. 368-379, 2002.

[2] D. Baleanu and J. J. Trujillo, "On exact solutions of a class of fractional Euler-Lagrange equations," Nonlinear Dynamics, vol. 52, no. 4, pp. 331-335, 2008.

[3] T. A. Maraaba, F. Jarad, and D. Baleanu, "On the existence and the uniqueness theorem for fractional differential equations with bounded delay within Caputo derivatives," Science in China A, vol. 51, no. 10, pp. 1775-1786, 2008.

[4] A. A. Kilbas, H. M. Srivastava, and J. J. Trujillo, Theory and Applications of Fractional Differential Equations, vol. 204, Elsevier Science, Amsterdam, The Netherlands, 2006.

[5] J. T. Machado, V. Kiryakova, and F. Mainardi, "Recent history of fractional calculus," Communications in Nonlinear Science and Numerical Simulation, vol. 16, no. 3, pp. 1140-1153, 2011.

[6] F. C. Meral, T. J. Royston, and R. Magin, "Fractional calculus in viscoelasticity: an experimental study," Communications in Nonlinear Science and Numerical Simulation, vol. 15, no. 4, pp. 939-945, 2010.

[7] K. B. Oldham and J. Spanier, The Fractional Calculus, Academic Press, New York, NY, USA, 1974.

[8] I. Podlubny, Fractional Differential Equations, vol. 198, Academic Press, New York, NY, USA, 1999.
[9] H. Weitzner and G. M. Zaslavsky, "Some applications of fractional equations," Communications in Nonlinear Science and Numerical Simulation, vol. 8, no. 3-4, pp. 273-281, 2003.

[10] Z. Bai and H. Lü, "Positive solutions for boundary value problem of nonlinear fractional differential equation," Journal of Mathematical Analysis and Applications, vol. 311, no. 2, pp. 495-505, 2005.

[11] G. Chai, "Positive solutions for boundary-value problem of fractional differential equation with $p$-Laplacian operator," Boundary Value Problems, vol. 2012, pp. 1-18, 2012.

[12] T. Chen and W. Liu, "An anti-periodic boundary value problem for the fractional differential equation with a $p$-Laplacian operator," Applied Mathematics Letters, vol. 25, no. 11, pp. 16711675, 2012.

[13] W. Feng, S. Sun, Z. Han, and Y. Zhao, "Existence of solutions for a singular system of nonlinear fractional differential equations," Computers \& Mathematics with Applications, vol. 62, no. 3, pp. 1370-1378, 2011.

[14] G. Wang, L. Zhang, and S. K. Ntouyas, "Existence of multiple positive solutions of a nonlinear arbitrary order boundary value problem with advanced arguments," Electronic Journal of Qualitative Theory of Differential Equations, no. 15, pp. 1-13, 2012.

[15] J. Wang, H. Xiang, and Z. Liu, "Existence of concave positive solutions for boundary value problem of nonlinear fractional differential equation with $p$-Laplacian operator," International Journal of Mathematics and Mathematical Sciences, vol. 2010, Article ID 495138, 17 pages, 2010.

[16] X. Xu, D. Jiang, and C. Yuan, "Multiple positive solutions to singular positone and semipositone Dirichlet-type boundary value problems of nonlinear fractional differential equations," Nonlinear Analysis: Theory, Methods \& Applications, vol. 74, no. 16, pp. 5685-5696, 2011.

[17] X. Xu, D. Jiang, and C. Yuan, "Multiple positive solutions for the boundary value problem of a nonlinear fractional differential equation," Nonlinear Analysis: Theory, Methods \& Applications, vol. 71, no. 10, pp. 4676-4688, 2009.

[18] X. Yang, Z. Wei, and W. Dong, "Existence of positive solutions for the boundary value problem of nonlinear fractional differential equations," Communications in Nonlinear Science and Numerical Simulation, vol. 17, no. 1, pp. 85-92, 2012.

[19] Y. Zhao, S. Sun, Z. Han, and M. Zhang, "Positive solutions for boundary value problems of nonlinear fractional differential equations," Applied Mathematics and Computation, vol. 217, no. 16, pp. 6950-6958, 2011.

[20] Y. Zhao, S. Sun, Z. Han, and Q. Li, “The existence of multiple positive solutions for boundary value problems of nonlinear fractional differential equations," Communications in Nonlinear Science and Numerical Simulation, vol. 16, no. 4, pp. 2086-2097, 2011.

[21] Y. Zhou, F. Jiao, and J. Li, "Existence and uniqueness for $p$-type fractional neutral differential equations," Nonlinear Analysis: Theory, Methods \& Applications, vol. 71, no. 7-8, pp. 2724-2733, 2009.

[22] S. Liang and J. Zhang, "Positive solutions for boundary value problems of nonlinear fractional differential equation," Nonlinear Analysis: Theory, Methods \& Applications, vol. 71, no. 11, pp. 5545-5550, 2009.

[23] H. Lu and Z. Han, "Existence of positive solutions for boundaryvalue problem of fractional differential equation with $p$ laplacian operator," American Jouranl of Engineering and Technology Research, vol. 11, pp. 3757-3764, 2011. 
[24] J. Wang and H. Xiang, "Upper and lower solutions method for a class of singular fractional boundary value problems with $p$ Laplacian operator," Abstract and Applied Analysis, vol. 2010, Article ID 971824, 12 pages, 2010.

[25] J. G. Dix and G. L. Karakostas, "A fixed-point theorem for S-type operators on Banach spaces and its applications to boundary-value problems," Nonlinear Analysis: Theory, Methods \& Applications, vol. 71, no. 9, pp. 3872-3880, 2009.

[26] J. Xu, Z. Wei, and W. Dong, "Uniqueness of positive solutions for a class of fractional boundary value problems," Applied Mathematics Letters, vol. 25, no. 3, pp. 590-593, 2012.

[27] T. Chen, W. Liu, and Z. Hu, "A boundary value problem for fractional differential equation with $p$-Laplacian operator at resonance," Nonlinear Analysis: Theory, Methods \& Applications, vol. 75, no. 6, pp. 3210-3217, 2012.

[28] X. Liu, M. Jia, and X. Xiang, "On the solvability of a fractional differential equation model involving the $p$-Laplacian operator," Computers \& Mathematics with Applications, vol. 64, no. 10, pp. 3267-3275, 2012.

[29] Z. Han, H. Lu, S. Sun, and D. Yang, "Positive solutions to boundary-value problems of $p$-laplacian fractional differential equations with a parameter in the boundary," Electronic Journal of Differential Equations, vol. 2012, pp. 1-14, 2012.

[30] K. Diethelm, The Analysis of Fractional Differential Equations, vol. 2004, Springer, Berlin, Germany, 2010.

[31] G. H. Hardy, J. E. Littlewood, and G. Pólya, Inequalities, Cambridge University Press, Cambridge, UK, 1988.

[32] D. J. Guo and V. Lakshmikantham, Nonlinear Problems in Abstract Cones, vol. 5, Academic Press, Orlando, Fla, USA, 1988.

[33] T. Kim, "Some identities for the Bernoulli, the Euler and the Genocchi numbers and polynomials," Advanced Studies in Contemporary Mathematics, vol. 20, no. 1, pp. 23-28, 2010.

[34] T. Kim and D. S. Kim, "A note on higher-order Bernoulli polynomials," Journal of Inequalities and Applications, vol. 2013, article 111, 2013.

[35] D. S. Kim and T. Kim, "Some identities of higher order Euler polynomials arising from Euler basis," Integral Transforms and Special Functions, vol. 24, no. 9, pp. 734-738, 2012.

[36] T. Kim, "Some identities on the $q$-integral representation of the product of several $q$-Bernstein-type polynomials," Abstract and Applied Analysis, vol. 2011, Article ID 634675, 11 pages, 2011.

[37] S. Araci, "Novel identities for $q$-Genocchi numbers and polynomials," Journal of Function Spaces and Applications, vol. 2012, Article ID 214961, 13 pages, 2012.

[38] J. J. Seo, "Certain results on the $q$-Genocchi numbers and polynomials," Journal of the Chungcheong Mathematical Society, vol. 26, no. 1, 2013. 


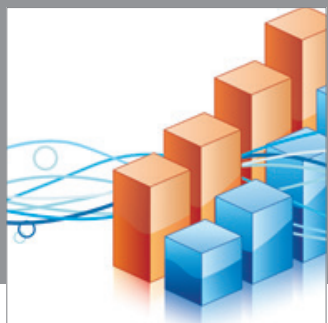

Advances in

Operations Research

mansans

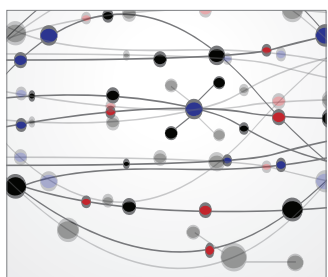

The Scientific World Journal
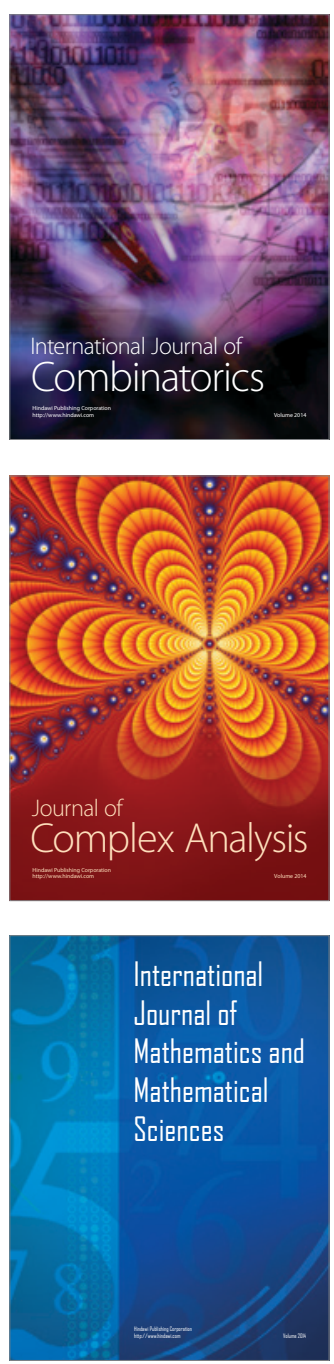
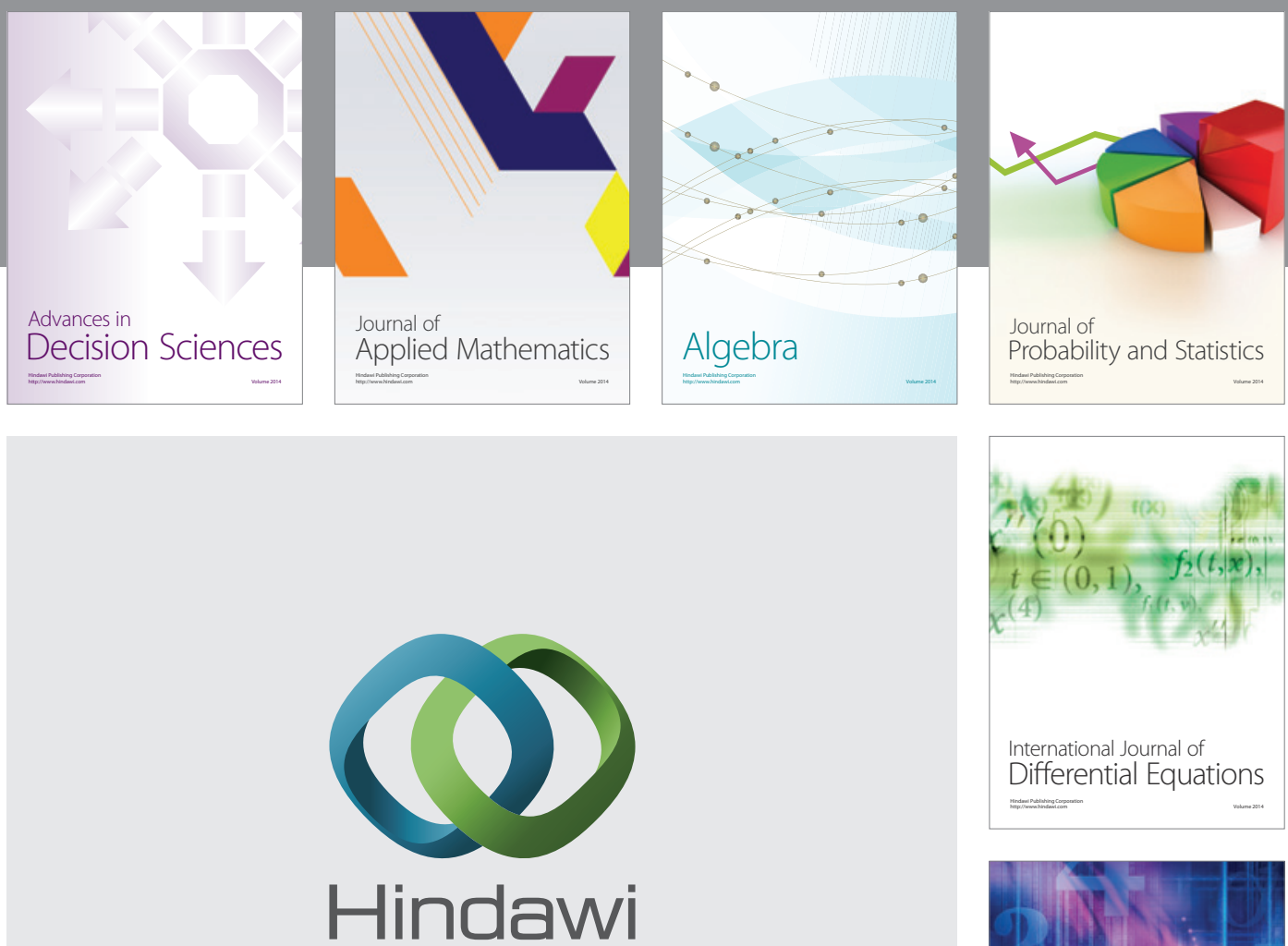

Submit your manuscripts at http://www.hindawi.com
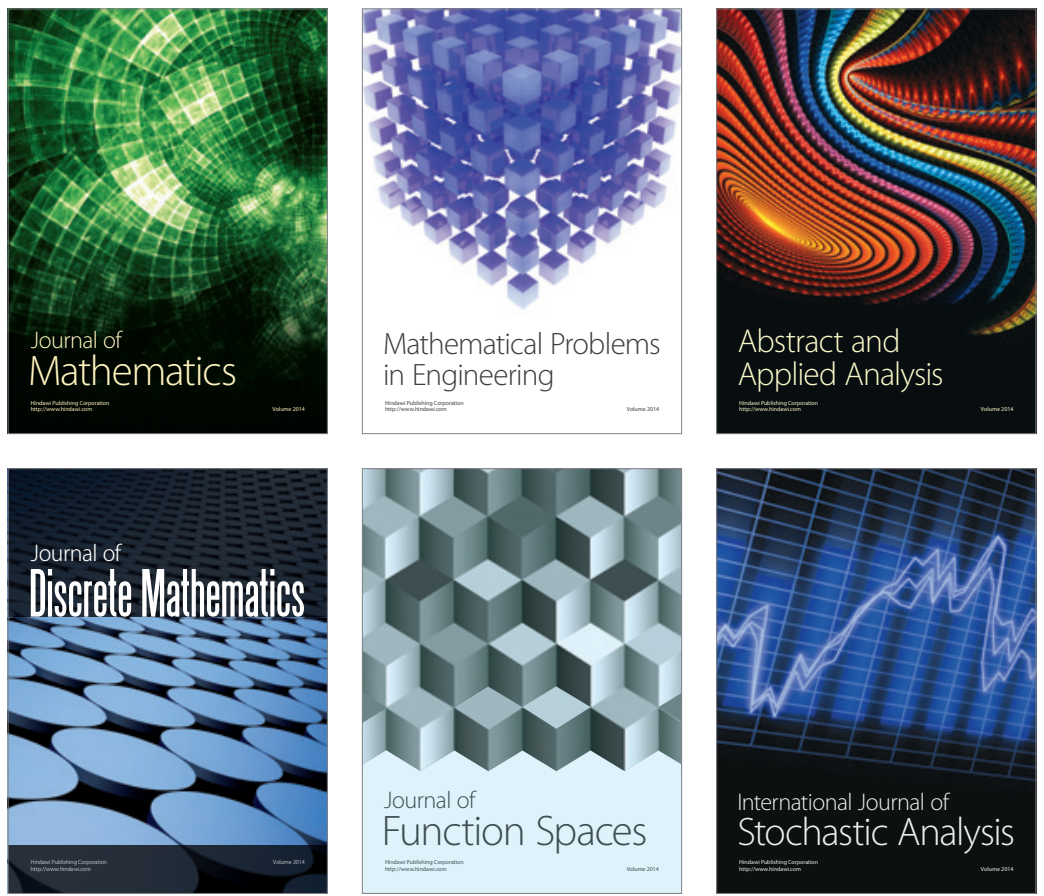

Journal of

Function Spaces

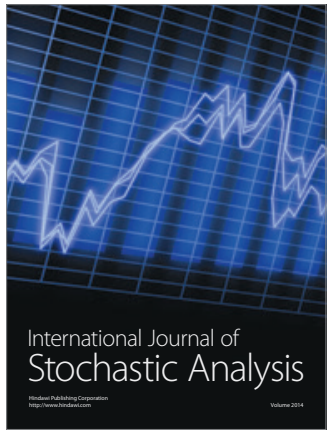

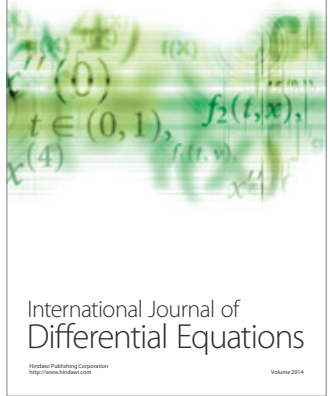
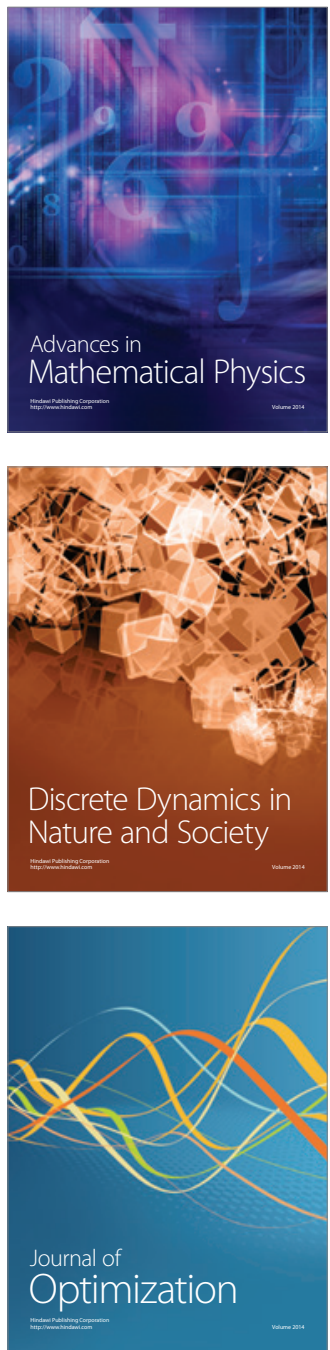